\title{
Socio-economic differences in psychiatric in-patient care
}

\author{
V. Lorant ${ }^{1}$, D. Kampfl², A. Seghers' 3 , D. Deliège ${ }^{1}$, M.-C. Closon¹, M. Ansseau² \\ ${ }^{1}$ Health System Research, School of Public Health, Faculty of Medicine, Université Catholique de Louvain, \\ Brussels, Belgium, \\ ${ }^{2}$ Department of Psychiatry and Medical Psychology, CHU, Université de Liège, Liège, Belgium and \\ ${ }^{3}$ Cliniques Saint Luc, Université Catholique de Louvain, Brussels, Belgium
}

KEYWORDS: socio-economic factors; hospitals; psychiatric; accessibility of health services; assessment; patient outcomes

\begin{abstract}
Objective: We seek to investigate socio-economic differences in psychiatric in-patient care regarding admission, treatment and outcome. Method: This study is undertaken on a comprehensive and exhaustive psychiatric case register of all psychiatric in-patient care carried out in Belgium in 1997 and 1998 (n 1/4 144 754). Results: Lower socio-economic groups were more likely to be compulsorily admitted, to be cared for in a non-teaching or psychiatric hospital, to be admitted in a hospital with unexpectedly long average length of stay and to be admitted to a ward with a more severe casemix. They were less likely to receive antidepressants and psychotherapies. The improvements in functioning and in symptoms were also less favourable for these groups. The lowest group had a higher risk of dying in the hospital. Conclusion: Psychiatric in-patient care is associated with moderate socio-economic differences in access, treatment and outcome. Further research is needed to clarify the causes of such disparities.
\end{abstract}




\section{Introduction}

The relationship between social class and mental disorder has been a longstanding issue in social psychiatry and psychiatric epidemiology $(1,2)$. Research has repeatedly shown a higher prevalence of mental disorders in the lower socio-economic groups. This socio-economic mental health gradient has been found in large-population epidemiological studies using psychiatric diagnostic instruments (3-5) or symptom checklists $(6,7)$. Although mental disorders remain largely untreated both in North America and Europe, several studies have evidenced few socioeconomic inequalities in mental care among subjects with psychiatric disorders (8).

Most previous studies addressing inequalities in mental care have assumed that a fair distribution of care is achieved when individuals of equal ill-health status use the same quantity of care, disregarding differences in quality of care and disparities in outcome (9). As far as mental health is concerned, however, there is some evidence of unequal quality and outcome of care. Lower socioeconomic groups use less specialized care (8), while medication use and dosage are also less appropriate in such groups (10). In addition, some population studies have shown that poverty and low socio-economic status (SES) increase the duration of episodes for a given baseline clinical status $(11,12)$. Among these small patient studies, some have evidenced poorer clinical course for low educational groups $(13,14)$.

There have also been some studies addressing socio-economic differences in out-patient or primary care $(8,15-19)$. But very few have addressed differences within in-patient psychiatric wards. Several pieces of research have attempted to predict the length of stay $(20,21)$. Others have evidenced a higher rate of psychiatric admission for individuals of low SES (22) or living in deprived areas $(23,24)$. Very few studies have addressed socio-economic disparities in admission, treatment and outcome of psychiatric in-patient care $(22,25)$.

Overall access to care in Belgium is relatively good. Social security grants $96.9 \%$ of the population with a health care insurance, an out-of-pocket health care expenditure share of $15 \%$ in average (26). It also has a high supply of general practitioners (GPs) and psychiatrists in the curative sector (respectively, 11.6 and 2.0 full-time equivalent per 10000 inhabitants). There is no referral gateway to the psychiatrist or to in-patient services. Moreover, patients have the option of choosing their hospital as there is no catchment area and because the country is small. Finally, Belgium has a higher density of psychiatric beds (1.7 per 1000 inhabitants) giving patients plenty of freedom to choose.

\section{AIMS OF THE STUDY}

This study addresses socio-economic disparities in psychiatric in-patient care. With the help of the comprehensive Psychiatric Case Register introduced in Belgium in 1996, we seek to assess socio-economic differences in admission, treatment and outcome of psychiatric in-patient care. 


\section{Material and methods}

\section{DATA}

Since 1996, all psychiatric admissions, treatments and discharges have been subject to mandatory registering in a unique format, the Minimum Psychiatric Summary (MPS). This psychiatric case register is a comprehensive record of all psychiatric in-patient activities covering admission, treatment and discharge (27). It is organized in 12 sections, containing 300 items. It covers information on the five DSM-IV axes, socio-economic background, previous living arrangements, referral process, ward and hospital identification, psychological symptoms at the beginning and at the end of the treatment, functioning at the beginning and at the end of the stay, evaluations, treatments and medication delivered. Each hospital has an MPS administrator, who controls the quality of the data. Training of the administrators and quality control are provided by a supervisor belonging to one of the seven participating universities.

In this study, we used the 166839 discharges occurring in the 1997-98 period; we excluded episodes of Psychiatric Community Care (PCC) or in psychogeriatric wards (n 1/4 1971); all episodes having their principal diagnosis defined on the second DSM-IV axis were excluded (n $1 / 4$ 17 674). After exclusion of missing values, we were left with 144754 discharges, accounting for $87 \%$ of total psychiatric discharges.

\section{DEFINITION OF VARIABLES}

Clinical status was assessed by the diagnosis on the five DSM-IV axes. The diagnoses on the first axis were divided into the following categories: substance-related disorders, mood disorders, schizophrenia and other psychotic disorders, dementia and other cognitive disorders, and others. The second axis disorders (presented as secondary diagnosis) were divided into personality disorders or mental retardation. In the third axis, the medical conditions were coded according to the ICD-9 and the number of somatic comorbidities was computed. For the fourth axis, we computed the number of psychosocial and environmental problems (28). The fifth axis is the Global Assessment and Functioning (GAF) scale, an instrument widely used to assess overall functioning on a continuum ranging from 1 to 100 (28). Lastly, the MPS used a scale of 29 items to register the presence of DSM-IV psychological symptoms such as suicide ideation, threats of auto-aggression, hostility, depressive mood, etc. We computed the number of symptoms.

Socio-economic status was defined by combining educational status, occupational level and activity status. Each individual was given a score equal to his or her mean relative ranking on these three variables. Individuals were then divided into five groups of rather equivalent size. As ties are more likely to occur, these groups do not strictly correspond to quintiles. Such a composite index has been previously used in psychiatric epidemiology (29). It helps to carry out a stratification on the whole lifecycle, integrating both early educational achievement and ulterior professional mobility.

Differences in psychiatric in-patient care were assessed for three main domains: admission setting, treatment received and the outcome of the stay. Five features indicating a less 
favourable admission were considered: mode of admission (compulsory vs. voluntary), the hospital type (psychiatric hospital vs. general hospital with a psychiatric ward, non-teaching hospital vs. teaching hospital), hospital performance in terms of length of stay (long-stay hospitals vs. normal-stay hospitals) and, finally, the average severity of the patients cared for in the ward to which the patient is admitted (average GAF score of the ward). Hospital performance was estimated by the Bosmans and Fecher method (30). Those hospitals in the lower 15 percentile (out of132) were classified as hospitals with unexpectedly long length of stay (HULLS).

For the three main groups of diagnosis, adequate treatments were defined according to current guidelines: antidepressants and psychotherapies for mood disorders, surveillance of withdrawal and relapse for substance-related disorders, and neuroleptic drugs for schizophrenic and other psychotic disorders (31). Four outcome variables were defined. The evolution of the GAF scale score (discharge score - admission score), the evolution in the number of psychological symptoms, readmission and in-hospital mortality rate.

\section{STATISTICAL ANALYSIS}

Generalized linear models (using GENMOD SAS procedure) were used to estimate the relationship between SES and the dependent variables. A logitlink function was used for the binary dependent variable (mode of admission, hospital type, treatment, readmission and mortality). All estimations were controlled for the five DSM-IV axis diagnoses, number of psychiatric and somatic comorbidities, number of psychological symptoms, previous psychiatric in-patient history, age and sex. Because the link between mental health and SES has been shown to be disorder-specific $(32,33)$, the risk factors are also presented for five main groups of mental disorders.

\section{Results}

Substance, mood and psychotic disorders accounted for three quarters of all discharges (see Table 1). Comorbidity was frequent, with more than one-third of the episodes having a personality disorder comorbidity, another axis-1 comorbidity as well as a somatic comorbidity. The most frequent diseases were epilepsy, diabetes, chronic liver cirrhosis, hypertension, obesity, cerebral degeneration and chronic bronchitis. On average, an episode presented 1.7 psychosocial and environmental problems. Overall GAF reached a score of 44 which is low compared with previous studies (34-38). A third of the patients discharged had a previous inpatient psychiatric history.

More than half of the episodes concerned adults between 35 and 64 years old. This was partly due to the exclusion of psychogeriatric wards, where most elderly patients are cared for. A small proportion of the episodes resulted from admission on a compulsory basis (11.9\%). Discharges were divided equally among general hospitals with psychiatric wards and psychiatric hospitals, with a small proportion of the episodes being cared for in teaching hospitals or in HULLS. Between admission and discharge, the GAF score improved by 12.9 (effect size $=0.82$ ) and the 
number of psychological symptoms was reduced by 1.4 (effect size $=-0.58$ ). Readmission and mortality rates reached 34 and $0.8 \%$, respectively.

Table 1. Clinical, sociodemographic and setting distribution of psychiatric inpatients

Percentage or mean

\section{Clinical status}

Diagnosis on axis $1(\%)$

Substance-related disorders

Mood disorders 27.9

Schizophrenia and other psychotic disorders 14.4

Dementia and other cognitive disorders 3.6

Others 20.8

Diagnosis on axis $2(\%)$

Personality disorder $\quad 37.7$

Mental retardation $\quad 4.2$

No diagnosis on axis $2 \quad 58.0$

Psychiatric comorbidity $(\%)^{*} \quad 36.0$

Physical comorbidities (\%) 26.9

Number of problems on axis 4 (mean and SE) $1.709(0.004)$

Global assessment of functioning (mean score and SE) $43.647(0.04)$

Having previous psychiatric history (\%) 36.2

Sociodemographic covariates

Sex $(\%)$

Men $\quad 51.2$

Women $\quad 48.8$

Age (\%)

$0-14 \quad 1.5$

$15-34 \quad 30.0$

$35-64 \quad 57.6$

$65+\quad 10.8$

Socio-economic status (\%)

First quintile 20.0

$\begin{array}{ll}\text { Second quintile } & 26.4\end{array}$

Third quintile 14.3

Fourth quintile $\quad 19.5$

Fifth quintile $\quad 19.8$

\section{Care setting}

Mode of admission (\%)

Voluntary

Mandatory

Teaching hospital (\%)

No

95.0

Yes

Hospital type (\%)

General hospital with a psychiatric ward 50.1

Psychiatric hospital

Hospital performance (\%):

Low

\section{Outcome}

GAF evolution score (discharge - admission)

$12.887(0.04)$

No. of psychological symptoms (discharge - admission)

$-1.388(0.01)$

Readmission (\%)

$33.8(0.1)$

Mortality (\%)

$0.8(0.02)$

* Onaxis 1 only. 
The socio-economic risk factors of unfavourable admission features, unexpected treatment and outcome are set out in Table 2. Each coefficient is controlled for axis-1 group disorders, axis-2 group disorders, number of psychiatric comorbidities, number of somatic comorbidities, number of psychosocial problems, admission GAF score, number of psychological symptoms, previous inpatient psychiatric history, age and sex. As SES increased, there was a slight monotonic decrease in the likelihood of being admitted mandatorily [first quintile, odds ratio $(\mathrm{OR})=1.22$ ]. Lower SES groups were more likely to be admitted to a nonteaching $(\mathrm{OR}=1.17)$ or psychiatric hospital $(\mathrm{OR}=1.47)$. Admission to HULLS also decreased with SES. A monotonic decreasing relationship was observed with respect to the overall functioning of patients in the ward: the higher the SES the better the mean GAF score of the ward where the patient was cared for. Hence, for most of the admission features, increasing SES was associated with more favourable circumstances.

Unexpected care was linked to SES. Among patients with mood disorders, the lowest SES group was more likely not to receive antidepressant drugs $(\mathrm{OR}=1.66)$ and psychotherapy $(\mathrm{OR}=$ 1.51). Such under-provision decreased monotonically with SES. Among patients with substance abuse-related disorders, a slight under-provision of withdrawal surveillance was observed for the lowest SES group (OR = 1.10). There were few socio-economic differences regarding the provision of neurotic drugs for psychotic patients.

Regarding outcome, there was a monotonically decreasing benefit from hospitalization as SES increased, with respect to GAF score and psychological symptoms. The lowest SES group improved its functioning to a lesser extent $(\beta=-3.57)$ and was left with more residual symptoms ( $\beta=0.22$ ) when compared with the highest group. The readmission rate was greater in the second-lower socio-economic group $(\mathrm{OR}=1.35)$. There was a significantly higher risk of mortality for the lowest SES group $(\mathrm{OR}=1.79)$. Mortality did not, however, present a monotonic decreasing relationship with SES.

The outcome differences were analysed further by groups of disorders (Table 3). In order to limit the number of coefficients, two SES groups were formed, dividing the sample into two groups of equal size. Again, all the results were controlled for the clinical and demographic variables presented previously. No unique ranking of the disorders was found. Considering GAF and the number of psychological symptoms, schizophrenia and substance disorders showed more unequal results, while affective disorders and dementia evidenced the least unequal distribution of outcome. Turning to readmission risk, the groups of disorders showed very similar socio-economic differences, except for dementia. The risk of mortality of the lower SES group was higher among the patients with substance-related disorders and other disorders.

Using educational level instead of the composite indicators led to slight differences (results not shown). Regarding admission, differences were more marked for some variables (use of nonteaching hospitals, admission to HULLS, severity of the patients cared in the same ward and non-psychotherapies) and smaller for other variables (compulsory admission, psychiatric hospitals and no antidepressant drugs). Outcome discrepancies were generally smaller when using educational level. 
Table 2. Risk of less favourable admission, of unexpected treatment and of outcome for psychiatric inpatient care: odds ratios (OR) and $\beta$ coefficient by socio-economic quintile

\begin{tabular}{|c|c|c|c|c|c|}
\hline & \multicolumn{5}{|c|}{ Socio-economic groups } \\
\hline & First quintile & Second quintile & Third quintile & Fourth quintile & Fifth quintile (ref) \\
\hline \multicolumn{6}{|l|}{ Unfavourable admission features } \\
\hline Mandatory admission (OR) & $1.22(0.019)^{* * *}$ & $1.21(0.017)^{* * *}$ & $1.08(0.02)^{* * *}$ & $1.05(0.018)^{* *}$ & 1 \\
\hline Non-teaching hospital (OR) & $1.17(0.029)^{* * *}$ & $1.10(0.026)^{* * *}$ & $1.21(0.029)^{* * *}$ & $1.08(0.028)^{* *}$ & 1 \\
\hline Psychiatric hospital (OR) & $1.47(0.044)^{* * *}$ & $1.50(0.04)^{* * *}$ & $1.31(0.045)^{* * *}$ & $1.33(0.041)^{* * *}$ & 1 \\
\hline Long-stay hospital (OR) & $1.2(0.027)^{* * *}$ & $1.10(0.026)^{* * *}$ & $1.21(0.029)^{* * *}$ & $1.08(0.028)^{* *}$ & 1 \\
\hline Severity of the cases in the ward $(\beta)$ & $-1.63(0.08)^{* * *}$ & $-1.21(0.075)^{* * *}$ & $-0.84(0.086)^{* * *}$ & $-0.27(0.079)^{* * *}$ & 0 \\
\hline \multicolumn{6}{|l|}{ Unexpected treatment } \\
\hline No antidepressant drug for patients with mood disorder (OR) & $1.66(0.042)^{* * *}$ & $1.23(0.041)^{* * *}$ & $1.16(0.048)^{* *}$ & $1.05(0.044)$ & 1 \\
\hline No psychotherapy for patients with mood disorder (OR) & $1.51(0.034)^{* * *}$ & $1.47(0.032)^{* * *}$ & $1.34(0.038)^{* * *}$ & $1.17(0.034)^{* * *}$ & 1 \\
\hline No withdrawal surveillance for patients with substance disorder (OR) & $1.10(0.032)^{* *}$ & $1.00(0.029)$ & $1.02(0.032)$ & $0.97(0.029)$ & 1 \\
\hline No neuroleptic drugs for psychotic patients (OR) & $0.98(0.077)$ & $0.85(0.077)^{*}$ & $1.01(0.086)$ & $1.01(0.085)$ & 1 \\
\hline \multicolumn{6}{|l|}{ Outcome } \\
\hline GAF evolution (discharge score - admission score) ( $\beta$ ) & $-3.57(0.12)^{* * *}$ & $-2.81(0.12)^{* * *}$ & $-1.94(0.13)^{* * *}$ & $-1.14(0.12)^{* * *}$ & 0 \\
\hline Symptom evolution (symptoms at discharge -symptoms at admission) ( $\beta$ ) & $0.22(0.02)^{* * *}$ & $0.17(0.02)^{* * *}$ & $0.14(0.02)^{* * *}$ & $0.1(0.02)^{* * *}$ & 0 \\
\hline Readmission (OR) & $1.04(0.03)$ & $1.35(0.03)^{* * *}$ & $1.21(0.03)^{* * *}$ & $1.16(0.03)^{* * *}$ & 1 \\
\hline Mortality (OR) & $1.79(0.15)^{* * *}$ & $1.32(0.15)$ & $0.96(0.18)$ & $1.19(0.16)$ & 1 \\
\hline
\end{tabular}

OR: multivariate odds ratio; $\beta$ : multivariate regression coefficient. Standard error of the estimate in parenthesis. Significance: ${ }^{*} 0.05,{ }^{* *} 0.01,{ }^{* * *} 0.001$. Each coefficient is controlled for axis- 1 group of disorders, axis- 2 group of disorders, number of psychiatric comorbidities, number of somatic comorbidities, number of psychosocial problems, GAF score, number of psychological symptoms, previous psychiatric history, age and sex.

Table 3. Socio-economic risk of less favourable outcome of psychiatric hospitalization by DSM groups: odds ratios and $b$ coefficients of the lower 50 percentile compared with the higher 50 percentile

\begin{tabular}{lccc}
\hline & Evolution of GAF score $\beta$ & Evolution of number of symptoms $\beta$ & Readmission OR \\
\hline Substance & $-2.09(0.14)^{* * *}$ & $0.07(0.02)^{* * *}$ & $1.09(0.03)^{* *}$ \\
Mood & $-1.30(0.15)^{* * *}$ & $0.09(0.02)^{* * *}$ & $1.17(0.03)^{* * *}$ \\
Schizophrenia & $-1.91(0.21)^{* * *}$ & $0.16(0.03)^{* * *}$ & $1.18(0.04)^{* * *}$ \\
Dementia & $-1.27(0.46)^{* *}$ & $0.12(0.07)$ & $1.35(0.13)^{*}$ \\
Others & $-1.73(0.18)^{* * *}$ & $0.04(0.02)$ & $1.06(0.18)$ \\
\hline
\end{tabular}

OR: multivariate odds ratio; $\beta$ : multivariate regression coefficient; standard error of the estimate in parenthesis.

Significance: ${ }^{*} 0.05,{ }^{* *} 0.01,{ }^{* * *} 0.001$. Each coefficient is controlled for axis-1 group of disorders, axis- 2 group of disorders, number of psychiatric comorbidities, number of somatic comorbidities, number of psychosocial problems, GAF score, number of psychological symptoms, previous psychiatric history, age and sex.

\section{Discussion}

Our results show that psychiatric hospitalization is associated with socio-economic differences in terms of access to more favourable settings, adequate treatment and outcome. First, the likelihood of availing more favourable settings for in-patient mental care increased with SES: lower SES individuals were less likely to be admitted to a teaching or general hospital, and they had a higher likelihood of being admitted through compulsory admission, of finding themselves in a more severe case-mix ward, and of being cared for in HULLS. Secondly, lower SES groups with mood disorders received less psychotherapy and fewer antidepressant drugs. Thirdly, we found evidence that lower SES groups achieved less improvement in their functioning and psychological symptoms. This suggests that psychiatric hospitalization is associated with low to moderate inequalities in mental care and health. 
The present study could be affected by three sources of bias, related to confounding, selfselection and accuracy. Although this study counts with several clinical variables, we did not have a severity score. There is, hence, a risk that severity may confound the relationship between SES and admission setting, treatment or outcome. Previous studies have suggested that lower SES patients presented more severe symptoms, yielding a less favourable outcome. For two reasons we feel rather confident that our estimations would not be strongly affected. First, our results are controlled for functioning, which is linked to severity; secondly, the level of difference was similar when comparing severe major depression (296.23) with moderate (296.22) or mild major depression (296.21), or when comparing alcohol abuse (305.00) with alcohol dependence (303.9). However, in the absence of a validated score (such as an Hamilton), the role of severity cannot totally be ruled out. Other sources of confounding could alter our results. Help-seeking behaviour, referral patterns and financial barriers could influence the inequalities in access. It is well known that individuals of lower SES use more GP whereas higher SES individuals lay emphasis on speciality mental health care (8). Preferences for psychotherapeutic approach in the treatment of mental health might also explain that higher SES groups received more psychotherapies (39). Ethnicity - a variable not registered in the MPS has also been shown to influence referral and help-seeking behaviour (40). Finally, family support, coping style and verbal IQ are other possible confounding factors that we do not count with. All these covariates might explain part of the socio-economic differences found in this study.

Self-selection could arise in this study. Psychiatric hospitalization concerns only a small proportion (around 5\%) of all psychiatric patients (41). Hence, the present findings cannot be extrapolated to all mental care, as they refer to more chronic, severe and comorbid disorders than those treated in ambulatory settings (41). Localization of the hospitals in urban centres could also favour a higher use by lower SES groups. However, it is unlikely that the SES gradient would be accounted for by the localization of hospitals. First, hospitals do not have catchment area and patients can roam freely between hospitals. Also, Belgium counts with a high density of psychiatric hospital within a small territory. Finally, most of the teaching hospitals or psychiatric wards of general hospitals are located in urban centres so that one type of hospital is not spatially more accessible than another type. Hence, we think that localization is unlikely to be a strong determinant of the observed inequalities.

A final self-selection issue stems from the lack of unique patient number, providing revolvingdoor or chronic patients with more weights. As such patients are over-represented, there is a risk of a slight overestimation of the inequalities because chronic patients generally have a lower SES. Although we have been able to stifle out part of such confounding factors by controlling for previous psychiatric hospitalization, there is still a risk of a slight overestimation of the inequalities because chronicity is more likely to be underestimated.

A final limitation of this study is due to the type of data used. This information is not registered by researchers and may lack accuracy, as has been shown by studies of discharge registers (42, 43). Although the MPS has never been used to capitate resources for psychiatric hospitals, many clinicians in fact feared such a prospect, which may have led to over-scoring of patients. Indeed, when focusing on GAF scoring we found a small interaction effect between diagnosis on the first four DSM axes and hospital, implying that some hospitals tend to score a higher or lower GAF for 
a given level on the first four DSM axes. Some previous studies have raised doubts about the validity and reliability of the GAF, particularly for measuring functioning (38). However, although any such unreliability may affect inter-hospital comparisons, it is unlikely to influence the socio-economic gradient observed in this study. Moreover, the GAF still belongs to the DSMIV multiaxial diagnosis and recent studies have shown that it performs well in comparison with alternative measures of functioning (44). Overall, the GAF appears to be reasonably valid, with moderate reliability $(36,45)$.

Our results are consistent with previous studies addressing socio-economic inequalities in outpatient care. A recent cross-national comparison study showed that higher socio-economic groups used more specialized care, while lower SES individuals used primary care providers (8, 18). Evidence of treatment differences, for a given setting, is slight. A recent study has shown that depression recognition by GPs was dependent on sociodemographic correlates $(46,47)$. There is also some indication that better-educated individuals favour a psychotherapeutic approach, whereas those in lower educational groups have a more favourable opinion of the use of medication for mental health problems (48). Regarding outcome inequalities, this study is consistent with the Hampshire depression results. This project showed that, for a given baseline score, employment status as well as area-level of deprivation predicted both remission and improvement of depression at 6 weeks and 6 months (49).

While most previous studies have focused on access to mental health care, our study suggests that differences do not apply only to access but also to treatment and outcome. In fact, differences in access are rather limited. The work done hints that socio-economic difference also arises in the way patients are cared for, once admitted in a psychiatric in-patient setting and in the outcome of psychiatric hospitalization.

This may have important implications for the financing and delivery of mental care and for clinical practice. First, the study provides a double rationale for a review of the current financing scheme. The unequal allocation of resources by hospital type entails an unequal distribution of resources for patients with equal needs. As higher SES groups are more able to use betterfinanced hospitals (teaching and general hospitals have better staff / bed ratios and drugs reimbursement), this implies that the current system promotes a regressive allocation of resources. This is particularly true for the new - and costly - atypical antipsychotic drugs (50): a patient admitted in better financed hospitals might be more likely to receive such drugs. However, even if hospital resource allocation were made according to clinical status, the resulting capitation system would still disregard the unequal capacity to the benefit of the lower socio-economic groups. As these groups exhibit not only a poorer health status but also a poorer prognosis, an allocation scheme based on ill-health status would still be less favourable to lower SES groups. In some countries, hospital resources are distributed according to the socioeconomic background of the population cared for, for a given clinical status (51). An explanation of SES within the allocation scheme would be one way of accounting for unequal ill-health status as well as unequal prognosis. Secondly, as this study has shown, achieving a better distribution of resources within the psychiatric in-patient setting will not eradicate differences. It is, thus, the delivery of psychiatric care as a whole that needs to be examined. Belgium has almost twice the average European supply of psychiatric beds (0.93 per 1000 inhabitants), with an emphasis on big psychiatric hospitals. This is not what the WHO has been advocating. Belgium is still far from 
the WHO recommendations of integrating mental health within the general sector, scrapping big psychiatric hospitals and developing community mental health services (52). Finally, the underprovision of psychotherapies to lower SES individuals in this in-patient setting can be a subject of worry. If psychotherapies are effective, then efforts should be developed to improve their clinical delivery to different SES groups. This issue should be given thorough attention by policy makers and clinicians in order to promote equal use of indicated treatment within psychiatric in-patient wards. Future research should also try to disentangle the demand-side factors (i.e. patient preferences, cognitive abilities, etc.) and supply side factors (patient/staff ratios, type of psychotherapies, financing rules and clinical bias) influencing differences in psychiatric inpatient care.

\section{Acknowledgements}

Fonds de la Recherche Fondamentale Collective, Grant No. 2.4586.000. Programme AGORA des Services Scientifiques, Techniques et Culturels. 


\section{References}

1. Dohrenwend BP, Dohrenwend BS. Social status and psychological disorder: a causal inquiry. New York, NY: John Wiley \& Sons, 1969.

2. Kohn R, Dohrenwend B, Mirotznik J. Epidemiological findings on selected psychiatric disorders in the general population. In: Dohrenwend B, ed. Adversity, stress and psychopathology. New York: Oxford University Press, 1998:235-284.

3. Kessler RC, McGonagle KA, Zhao SY et al. Lifetime and 12-month prevalence of DSM-III-R psychiatric disorders in the United States - results from the national comorbidity survey. Arch Gen Psychiatry 1994;51:8-19.

4. Lewis G, Bebbington P, Brugha T et al. Socioeconomic status, standard of living, and neurotic disorder. Lancet 1998;352:605-609.

5. Bijl RV, Ravelli A, Van Zessen G. Prevalence of psychiatric disorder in the general population: results of The Netherlands Mental Health Survey and Incidence Study (NEMESIS). Soc Psychiatry Psychiatr Epidemiol 1998;33:587-595.

6. Reijneveld SA, Schene AH. Higher prevalence of mental disorders in socioeconomically deprived urban areas in the Netherlands: community or personal disadvantage? J Epidemiol Community Health 1998;52:2-7.

7. Weich S, Lewis G. Material standard of living, social class, and the prevalence of the common mental disorders in Great Britain. J Epidemiol Community Health 1998;52:8-14.

8. Alegria M, Bijl RV, Lin E, Walters EE, Kessler RC. Income differences in persons seeking outpatient treatment for mental disorders: a comparison of the United States with Ontario and The Netherlands. Arch Gen Psychiatry 2000;57:383-391.

9. Goddard M, Smith P. Equity of access to health care services: theory and evidence from the UK. Soc Sci Med 2001;53:1149-1162.

10. Young AS, Klap R, Sherbourne CD, Wells KB. The quality of care for depressive and anxiety disorders in the United States. Arch Gen Psychiatry 2001;58:55-61.

11. Weich S, Lewis G.Poverty, unemployment, and common mental disorders: population based cohort study. BMJ 1998;317:115-119.

12. Sargeant JK, Bruce ML, Florio LP, Weissman MM. Factors associated with 1-year outcome of major depression in the community. Arch Gen Psychiatry 1990;47:519-526.

13. Ronalds C, Creed F, Stone K, Webb S, Tomenson B. Outcome of anxiety and depressive disorders in primary care. Br J Psychiatry 1997;171:427-433.

14. Swindle RW JR, Cronkite RC, Moos RH. Risk factors for sustained nonremission of depressive symptoms: a 4-year follow-up. J Nerv Ment Dis 1998;186:462-469.

15. Cooper-Patrick L, Crum RM, Ford DE. Characteristics of patients with major depression who received care in general medical and specialty mental-health settings. Med Care 1994;32:15-24.

16. Fortney J, Rost K, Zhang M. A joint choice model of the decision to seek depression treatment and choice of provider sector. Med Care 1998;36:307-320. 
17. Katz SJ, Kessler RC, Frank RG, Leaf P, Lin E. Mental health care use, morbidity, and socioeconomic status in the United States and Ontario. Inquiry 1997;34:38-49.

18. Ten Have M, Vollebergh W, Bijl RV, De Graaf R. Predictors of incident care service utilisation for mental health problems in the Dutch general population. Soc Psychiatry Psychiatr Epidemiol 2001;36:141-149.

19. Tweed DL, Goering P, Lin E, Williams JI. Psychiatric morbidity and physician visits: lessons from Ontario. Med Care 1998;36:573-585.

20. Goldney RD, Fisher LJ, Walmsley SH. The Health of the Nation Outcome Scales in psychiatric hospitalisation: a multicentre study examining outcome and prediction of length ofstay. Aust NZ J Psychiatry 1998;32:199-205.

21. Huntley DA, Cho DW, Christman J, Csernansky JG. Predicting length of stay in an acute psychiatric hospital. Psychiatr Serv 1998;49:1049-1053.

22. Aro S, Aro H, Salinto M, Keskimaki I. Educational level and hospital use in mental disorders - a population-based study. Acta Psychiatr Scand 1995;91:305-312.

23. Dekker J, Peen J, Goris A, Heijnen H, Kwakman H. Social deprivation and psychiatric admission rates in Amsterdam. Soc Psychiatry Psychiatr Epidemiol 1997;32:485-492.

24. Driessen G, Gunther N, Van Os J. Shared social environment and psychiatric disorder: a multilevel analysis of individual and ecological effects. Soc Psychiatry Psychiatr Epidemiol 1998;33:606-612.

25. Muntaner C, Wolyniec P, McGrath J, Pulver AE. Psychotic inpatients social-class and their 1st admission to state or private psychiatric baltimore hospitals. Am J Public Health 1994;84:287-289.

26. Health Data. Paris: CREDES-OECD, 2000.

27. Cellule Psychiatrie. Manuel pour l'enregistrement du Résumé Psychiatrique Minimum. Bruxelles: Ministère des Affaires Sociales et de la Santé Publique, 1997.

28. American Psychiatric Association. DDM IV. Manuel diagnostique et statistique des troubles mentaux. Paris: Masson, 1996.

29. Regier DA, Farmer RE. One-month prevalence of mental disorders in the United-States and Sociodemographic characteristics: the Epidemiologic Catchment Area Study. Acta Psychiatr Scand 1993;88:35-47.

30. Bosmans N, Fecher F. Performance of Belgian hospitals: a frontier approach. Health Econ 1995;4:389397.

31. American Psychiatric Association. Practice guidelines for the treatment of psychiatric disorders: compendium 20002000.

32. Dohrenwend BP, Levav I, Shrout PE et al. Socioeconomic status and psychiatric disorders: the causation-selection issue. Science 1992;255:946-952.

33. Johnson JG, Cohen P, Dohrenwend BP, Link BG, Brook JS. A longitudinal investigation of social causation and social selection processes involved in the association between socioeconomic status and psychiatric disorders. J Abnorm Psychol 1999;108:490-499.

34. Segal SP, Egley L, Watson MA, Goldfinger SM. The quality of psychiatric emergency evaluations and patient outcomes in county hospitals. Am J Public Health 1995;85:1429-1431.

35. Tibbo P, Joffe K, Chue P, Metelitsa A, Wright E. Global assessment of functioning following assertive community treatment in Edmonton, Alberta: a longitudinal study. Can J Psychiatry Revue Can Psychiatrie 2001;46:144-148. 
36. Jones SH, Thornicroft G, Coffey M, Dunn G. A brief mental health outcome scale - reliability and validity of the Global Assessment of Functioning (GAF). Br J Psychiatry 1995;166:654-659.

37. Alaja R, Tienari P, Seppa K et al. Patterns of comorbidity in relation to functioning (GAF) among general hospital psychiatric referrals. European Consultation-Liaison Workgroup. Acta Psychiatr Scand 1999;99:135-140.

38. Moos RH, McCoy L, Moos BS. Global assessment of functioning (GAF) ratings: determinants and role as predictors of one-year treatment outcomes. J Clin Psychol 2000;56:449-461.

39. Jorm AF, Christensen H,Medway J,Korten AE, Jacomb PA, Rodgers B. Public belief systems about the helpfulness of interventions for depression: associations with history of depression and professional helpseeking [In Process Citation]. Soc Psychiatry Psychiatr Epidemiol 2000;35:211-219.

40. Ten Have ML, Bijl RV. Inequalities in mental health care and social services utilisation by immigrant women. Eur J Public Health 1999;9:45-51.

41. Bijl RV, Ravelli A. Psychiatric morbidity, service use, and need for care in the general population: results of The Netherlands Mental Health Survey and Incidence Study. Am J Public Health 2000;90:602607.

42. Vergnon P, Morgon E, Dargent S et al. Quality assessment of the medical information of the standardised discharge abstract. Revue d'Epidémiologie de Santé Publique 1998;46:24-33.

43. Calle JE, Saturno PJ, Parra P et al. Quality of the information contained in the minimum basic data set: Results from an evaluation in eight hospitals. Eur J Epidemiol 2000;16:1073-1080.

44. Hilsenroth MJ, Ackerman SJ, Blagys MD et al. Reliability and validity of DSM-IV axis V. Am J Psychiatry 2000;157:1858-1863.

45. Goldman HH, Skodol AE, Lave TR. Revising axis V for DSM-IV: a review of measures of social functioning. Am J Psychiatry 1992;149:1148-1156.

46. Kessler D, Lloyd K, Lewis G, Gray DP. Cross sectional study of symptom attribution and recognition of depression and anxiety in primary care. BMJ 1999;318:436-439.

47. Odell SM, Surtees PG, Wainwright NW, Commander MJ, Sashidharan SP. Determinants of general practitioner recognition of psychological problems in a multi-ethnic inner-city health district. $\mathrm{Br} \mathrm{J}$ Psychiatry 1997;171:537-541.

48. Jorm AF, Korten AE, Jacomb PA, Christensen H, Rodgers B, Pollitt P. Public beliefs about causes and risk factors for depression and schizophrenia. Soc Psychiatry Psychiatr Epidemiol 1997;32:143-148.

49. Ostler K, Thompson C, Kinmonth ALK, Peveler RC, Stevens L, Stevens A. Influence of socio-economic deprivation on the prevalence and outcome of depression in primary care - The Hampshire Depression Project. Br J Psychiatry 2001;178:12-17.

50. Lyons D, McLoughlin DM. Recent advances: psychiatry. BMJ 2001;323:1228-1231.

51. Smith P, Sheldon TA, Carr Hill RA, Martin S, Peacock S, Hardman G. Allocating resources to health authorities: results and policy implications of small area analysis of use of inpatient services. BMJ 1994;309:1050-1054.

52. Organisation Mondiale De La Santé. Rapport sur la santé dans le monde 2001. La santé mentale: nouvelle conception, nouveaux espoirs. Genève: OMS, 2001. 